\title{
Extension of DAMAS Phased Array Processing for Spatial Coherence Determination (DAMAS-C)
}

\author{
Thomas F. Brooks* and William M. Humphreys, Jr. ${ }^{\dagger}$ \\ NASA Langley Research Center, Hampton, VA. 23681
}

The present study reports a new development of the DAMAS microphone phased array processing methodology that allows the determination and separation of coherent and incoherent noise source distributions. In 2004, a Deconvolution Approach for the Mapping of Acoustic Sources (DAMAS) was developed which decoupled the array design and processing influence from the noise being measured, using a simple and robust algorithm. In 2005, three-dimensional applications of DAMAS were examined. DAMAS has been shown to render an unambiguous quantitative determination of acoustic source position and strength. However, an underlying premise of DAMAS, as well as that of classical array beamforming methodology, is that the noise regions under study are distributions of statistically independent sources. The present development, called DAMAS-C, extends the basic approach to include coherence definition between noise sources. The solutions incorporate cross-beamforming array measurements over the survey region. While the resulting inverse problem can be large and the iteration solution computationally demanding, it solves problems no other technique can approach. DAMAS-C is validated using noise source simulations and is applied to airframe flap noise test results.

\section{SYMBOLS}

$a_{m} \quad$ shear layer refraction amplitude correction for $e_{m n}$

$\mathbf{A}_{C} \quad$ DAMAS-C matrix with $A_{n_{0} n, n_{0}^{\prime} n^{\prime}}$ components

$A_{n_{0} n, n_{0}^{\prime} n^{\prime}}$ reciprocal influence of cross-beamforming characteristics between grid points

$B \quad$ array half-power "beamwidth" of $3 \mathrm{~dB}$ down from beam peak maximum

$c_{0} \quad$ speed of sound in medium in the absence of mean flow

CSM cross spectral matrix

$\overline{\gamma_{n_{0} n}^{2}} \quad$ coherence between sources at $n_{0}$ and $n$

DR diagonal removal of $\mathbf{G}$ in array processing

$\mathbf{e}_{n} \quad$ steering vector for array for focus at grid point $n$

$e_{m n} \quad$ component of $\mathbf{e}_{n}$ for microphone $m$

$f \quad$ frequency

$\Delta f \quad$ frequency bandwidth resolution of spectra

$G_{m m^{\prime}} \quad$ cross-spectrum between $P_{m}$ and $P_{m^{\prime}}$

G matrix (CSM) of cross-spectrum elements $G_{m m^{\prime}}$

$H \quad$ height of chosen scan plane

$i \quad$ iteration number

$m \quad$ microphone identity number in array

$m^{\prime} \quad$ same as $m$, but independently varied

\footnotetext{
*Senior Research Scientist, Aeroacoustics Branch, Fellow AIAA.

${ }^{\dagger}$ Senior Research Scientist, Aeroacoustics Branch, Senior Member AIAA.
} 


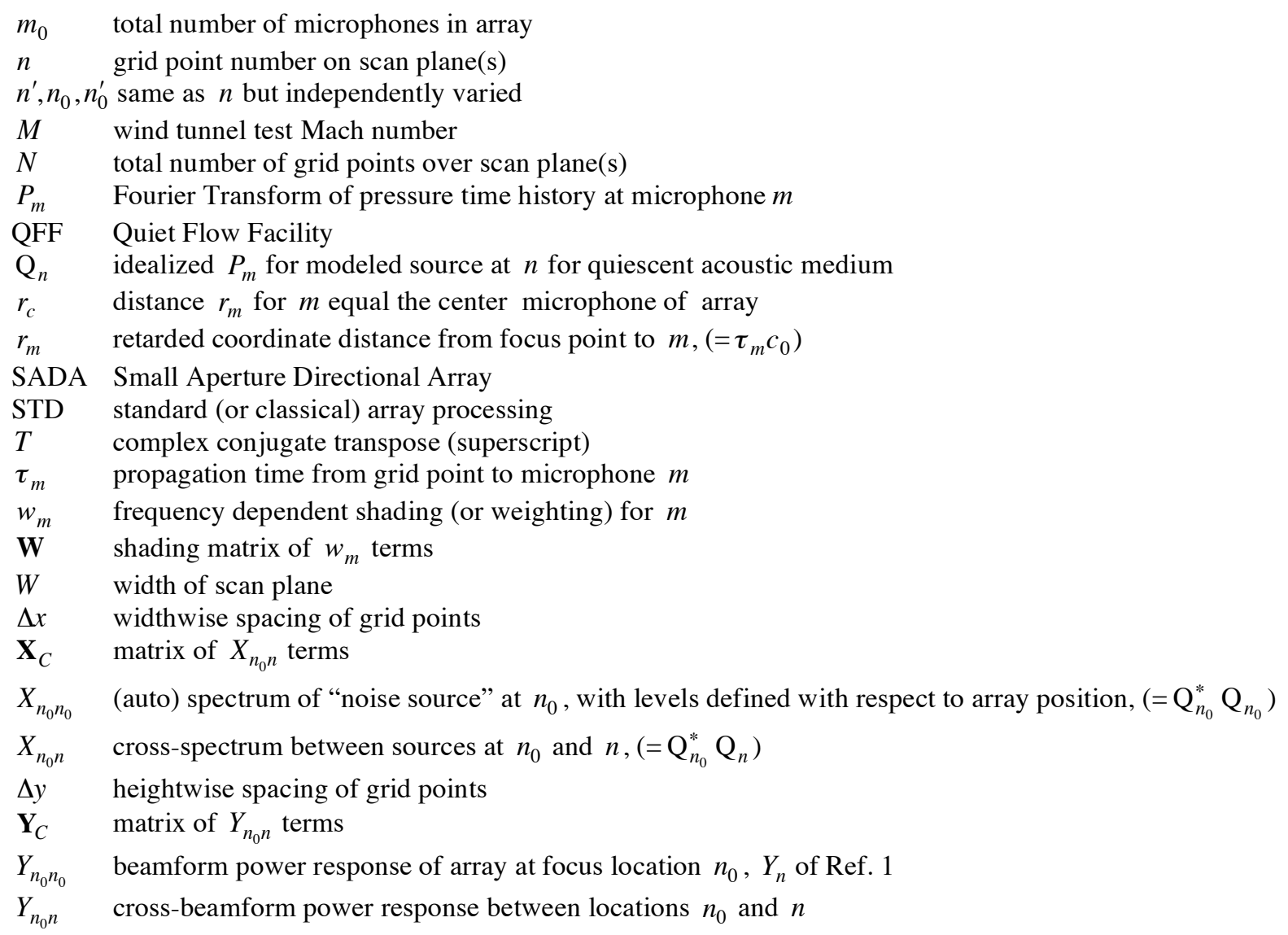

\section{INTRODUCTION}

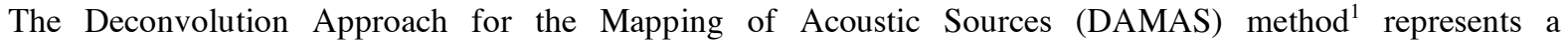
breakthrough in phased array processing technology that can remove array-dependent beamforming characteristics from output presentations. Whereas traditional beamforming produces an output that is dependent on array geometry, size, source distance, and frequency, DAMAS can remove these dependencies to render an accurate and explicit definition of acoustic source strength and location. Applications of DAMAS shown in Ref. 1 demonstrated its ability to provide quantitative airframe noise definition that was previously unattainable. The DAMAS algorithm is an iterative non-negative least squares solver of a linear system of equations (defining the DAMAS inverse problem) that is formulated based on the array response to distributions of modeled noise sources ${ }^{1,2}$. Threedimensional applications were examined in Ref. 3. Dougherty ${ }^{4}$ proposed two extensions to DAMAS. These were successfully applied to distributed source simulations and wake vortex turbulence decay noise data from flight tests. One extension termed DAMAS 2 offers dramatic speedups of the iteration calculations and adds solution regularization by a low pass filter. The other extension, DAMAS 3, does these and additionally reduces the required number of iterations. Both extensions in Ref. 4 restrict the point-spread function (array beamform lobe characteristics) to a translationally-invariant convolution form. This can be a serious limitation in spatial generality, but this is addressed through spatial transforms. DAMAS ${ }^{1}$ does not have such limitation concerns, although it is slower computationally. More recently, Dougherty applied the DAMAS methodology with success to turbofan engine duct mode measurement analysis ${ }^{5}$.

The underlying premise of classical beamform processing methodology (as does DAMAS) is the assumption that the source regions under study are distributions of statistically independent noise sources. This assumption is the basis for source power integration methodology, for example see Ref. 6. Classical beamforming produces insufficient information to determine how (and even if) source regions may be correlated. Normally one may suspect coherence may be present when the beamformed maps are "peculiar". Horne, et al. ${ }^{7}$ studied the effects of source coherence on array response for simple distributions of noise sources. It was found that distributed, coherent sources 
will radiate multi-lobed interference patterns with integrated source levels depending on array size and frequency. An expression for computing two-point cross beamforming over the array measurement scan plane was given and was suggested as a possibly useful spatial coherence diagnostic. Oerlemans and Sijtsma ${ }^{8}$ employed such an expression to perform coherence analyses between beamforming locations for reflections and aeroacoustic sources. The array outputs for simulation sources well matched experimental data for known sources. Still, from a source definition standpoint, the array beamforming coherence analysis approach is indeterminate for unknown source distributions. It was suggested ${ }^{7}$ that this cross beamforming coherence approach, combined with other knowledge, offers potential for the determination of coherence length of distributed sources and for the identification of sidelobes and mirror sources. A possible alternate approach to determine coherent source distributions using a microphone array is alluded to in Ref. 9. This would be a generalization of an array technique known as matched field processing. It would find combinations of point sources in the region that would coherently sum to the array cross-spectral matrix (CSM) eigenvectors. At present, there are no developed methods reported.

DAMAS, along with classical beamforming processing, employs the statistically independent (incoherent) noise source distribution assumption. It can thus produce inaccurate and distorted results in the presence of coherent sources. The original intent of the present study was to investigate the effect of source coherence on DAMAS processing and to develop methods to interpret and minimize error. It became apparent that it would be difficult to develop systematic diagnostic methods because of the complexity that coherence can cause in standard beamforming and DAMAS. During the study, a break came which offered the opportunity to generalize DAMAS to account for coherence, rather than just trying to identify and correct for it. In developing simulations of coherent sources in the noise field under study by DAMAS, it became apparent that an equation form similar to that of DAMAS can be rendered when coherence is allowed between sources and one employs cross-beamforming over the source region. This meant that if coherences between sources could be regarded as independent variables, then the cross-products between noise contributions of the sources are also independent variables. A DAMAS type linear system and solution should then be appropriate to determine a coherent as well as an incoherent distribution of sources. This paper presents the DAMAS-C (Coherence) development and some sample simulation and experimental applications.

\section{DAMAS-C ANALYSIS}

Beamforming and Cross-beamforming. Figure 1 shows a distribution of microphones comprising a phased array used to survey (or scan) noise regions. A scan plane with $N$ grid points is shown which cuts through a noise source region under study. Additional scan planes or individual grid points can also be placed over the source region when performing three-dimensional (3D) beamforming. For classical or standard (STD) array beamforming, the output power spectrum (or response) of the array is obtained from (using the terminology from Ref. 1)

$$
Y_{n}=\frac{\mathbf{e}_{n}^{T} \mathbf{G} \mathbf{e}_{n}}{m_{0}^{2}}
$$

where matrices are signified by bold letters. This is Eq. (6) from Ref. 1, where here $Y_{n}=Y(\mathbf{e})$, when focused at grid point $n$. The Cross-Spectral Matrix (CSM) is $\mathbf{G}$, where

$$
\mathbf{G}=\left[\begin{array}{cccc}
G_{11} & G_{12} & \cdots & G_{1 m_{0}} \\
\vdots & G_{22} & & \vdots \\
\vdots & & \ddots & \vdots \\
G_{m_{0} 1} & & & G_{m_{0} m_{0}}
\end{array}\right]
$$

where $m_{0}$ is the total number of microphones in the array. The steering "vector" matrix with respect to a

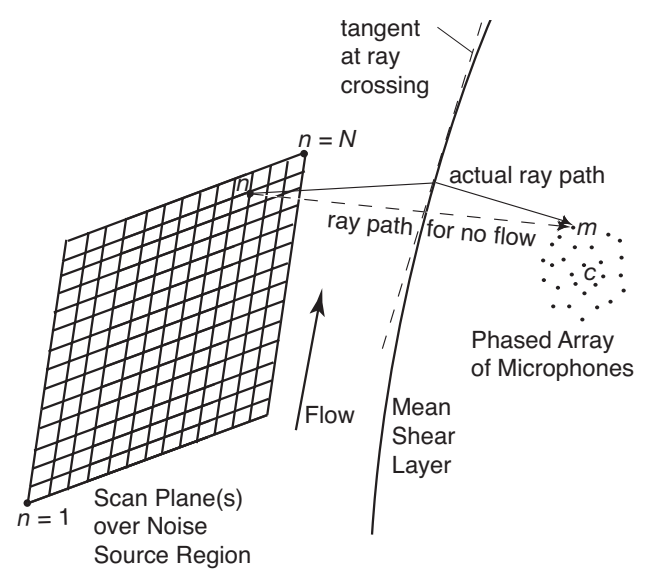

Figure 1. Illustration of open test configuration where the microphone array is placed outside the flow region containing the noise source scan plane region. 
survey grid point at location $n$ is

$$
\mathbf{e}_{n}=\operatorname{col}\left[\begin{array}{llll}
e_{1} & e_{2} & \cdots & e_{m_{0}}
\end{array}\right]
$$

and the component for each microphone $m$ is

$$
e_{m}=a_{m} \frac{r_{m}}{r_{c}} \exp \left(j 2 \pi f \tau_{m}\right)
$$

In Ref. 1, Eqs. (2), (3), and (4) correspond to the same equation numbers above. Figure 1 represents the test condition of an open jet test section with a noise region under study in the jet flow and the microphone array on the outside with a shear layer in between. The steering "vector" $e_{m}$ terms account for the mean amplitude $a_{m}$ and phase changes due to the convection and refraction through the shear layer to each microphone $m$. The time delay from a grid point to a microphone $m$ is $\tau_{m}$. In retarded coordinates, the distance from a point on the scan plane to the microphone $m$ is $r_{m}$ and the distance to the reference center microphone is $r_{c}$.

For the present analysis, we employ a crossbeamform product

$$
Y_{n_{0} n}=\frac{\mathbf{e}_{n_{0}}^{T} \mathbf{G} \mathbf{e}_{n}}{m_{0}^{2}}
$$

that is a beamform cross-spectrum (or cross-response) of the array between focused locations of grid points at $n=n_{0}$ and at another $n$. Equation (5) becomes Eq. (1) when $n_{0}=n$. For the steering "vectors" of Eq. (5),

$$
\mathbf{e}_{n_{0}}=\operatorname{col}\left[\begin{array}{llll}
e_{1 n_{0}} & e_{2 n_{0}} & \cdots & e_{m_{0} n_{0}}
\end{array}\right]
$$

and

$$
\mathbf{e}_{n}=\operatorname{col}\left[\begin{array}{llll}
e_{1 n} & e_{2 n} & \cdots & e_{m_{0} n}
\end{array}\right]
$$

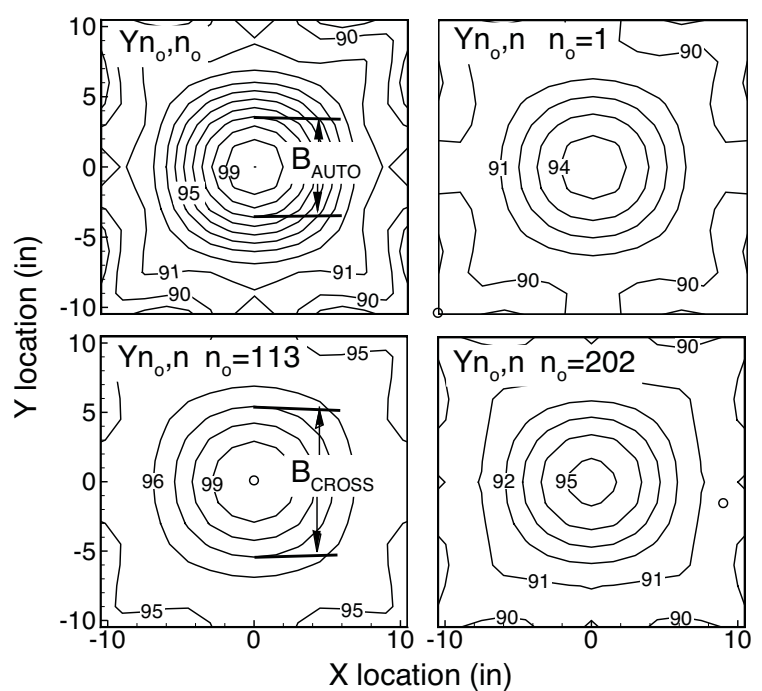

Figure 2. Output dB level contours over scan planes of (auto) Beamforming, $Y_{n_{0} n_{0}}$ and cross beamforming $Y_{n_{0} n}$ between grid points at $n_{0}$ and at $n$. Point source located at $n=113$.

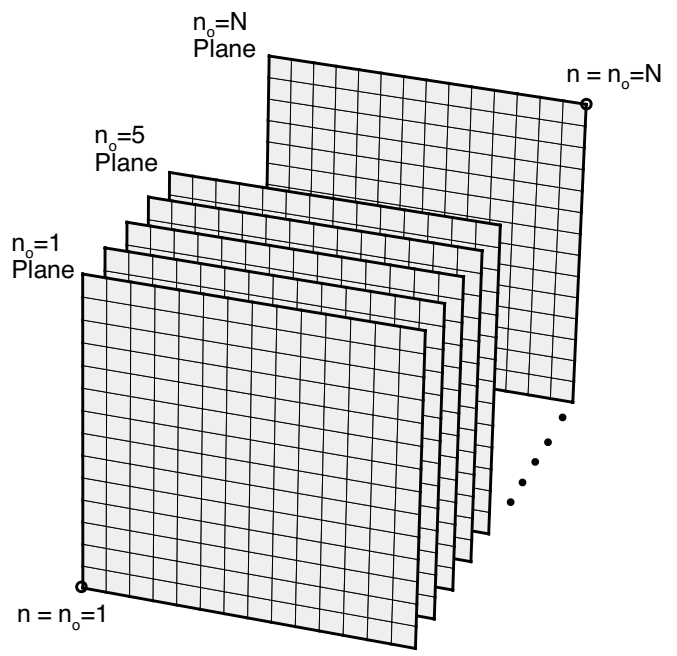

Figure 3. Illustration of stack of individual $n_{0}$ planes defining the $Y_{n_{0} n}$ survey and $X_{n_{0} n}$ solution space.

Figure 2, in the top left frame, shows a standard (auto) beamform dB contour map of $Y_{n_{0} n_{0}}$ for a simulated point source in the center of a $15 \times 15$ point grid scan plane (the focus points $n_{0}$ range from 1 to $N=225$ ). The array used is that of the SADA reported in Ref. 1 and detailed subsequently. Whereas in Ref. 1, all presentations were of beamforming and DAMAS solutions over the scan plane of $N$ points, the present paper often presents results over individual $n_{0}$ planes with grid points $n=1,2,3, \ldots, N$. Figures 2 present cross-beamform maps for the specific $n_{0}=1,113$, and 202 planes, respectively. The simulated source location is at $n=113$. In these planes, the respective $n_{0}$ grid locations are indicated by an open symbol. (Noted for subsequent use is that a length measure of the halfpower beamwidth $B$ is shown for $Y_{n_{0} n_{0}}$ and for a cross-beamform case $Y_{n_{0} n}$.) Figure 3 illustrates all $N$ of the $n_{0}$ planes. Each $n_{0}$ plane contains all cross-beamform responses $Y_{n_{0} n}$ over $n=1,2,3, \ldots, N-$ which includes its standard beamform response $Y_{n_{0} n_{0}}$ at $n_{0}$. 
DAMAS-C Inverse Problem Definition. From Ref. 1, Eq. (10), the pressure transform $P_{m}$ of microphone $m$ is related to a modeled source located at position $n$ in the source field.

$$
P_{m n}=\mathrm{Q}_{n} e_{m n}^{-1}
$$

where here, we have used the terminology $P_{m n}=\mathrm{Q}_{n} e_{m n}{ }^{-1}$ rather than $P_{m: n}=\mathrm{Q}_{n} e_{m: n}{ }^{-1}$ from Ref. 1. In Eq. (8), $\mathrm{Q}_{n}$ represents the pressure transform that $P_{m n}$ would be if flow convection and shear layer refraction did not affect transmission of the noise to microphone $m$ and if $m$ were at a distance of $r_{c}$ from $n$ rather than $r_{m}$. The $e_{m n}{ }^{-1}$ term is, from Ref. 1, those things that are postulated to affect the signal in the radiation transmission to render $P_{m n}$ (this is a way to back out $\mathrm{Q}_{n}$ information from $P_{m}$ ). For a single source located at $n$, Eqs. (11) and (12) of Ref. 1 gives relations for $P_{m n}{ }^{*} P_{m^{\prime} n}$ and $\mathbf{G}_{n_{\text {mod }}}$.

In the present analysis, it is desired to obtain a more general distribution for the CSM than that of a distribution of uncorrelated acoustic sources at different $n$, as given in Ref. 1. From Eq. (8), the cross-spectrum between microphones $m$ and $m^{\prime}$ for a distribution of sources over all $N$ grid points is

$$
P_{m}^{*} P_{m^{\prime}}=\sum_{n_{0}^{\prime}} \sum_{n^{\prime}}\left(\mathrm{Q}_{n_{0}^{\prime}} e_{m n_{0}^{\prime}}^{-1}\right)^{*}\left(\mathrm{Q}_{n^{\prime}} e_{m^{\prime} n^{\prime}}^{-1}\right)
$$

This reflects the fact that the acoustic pressure perceived at microphone $m$ due to the sources at $n_{0}^{\prime}$ and $n^{\prime}$ are generally different than that perceived at microphone $m^{\prime}$ for these same sources. Note here that $n_{0}, n, n_{0}^{\prime}$, and $n^{\prime}$ are distinguished from each other only in that they are varied separately.

As in Ref. 1, the $G_{m m^{\prime}}$ terms of the CSM are proportional to the corresponding $P_{m}{ }^{*} P_{m^{\prime}}$ terms. One has

$$
G_{m m^{\prime}}=\sum_{n_{0}^{\prime}} \sum_{n^{\prime}} X_{n_{0}^{\prime} n^{\prime}}\left(e_{m n_{0}^{\prime}}^{-1}\right)^{*} e_{m^{\prime} n^{\prime}}^{-1}
$$

where $X_{n_{0}^{\prime} n^{\prime}}=\mathrm{Q}_{n_{0}^{\prime}}^{*} \mathrm{Q}_{n^{\prime}}$. Upon accounting for FFT data ensemble processing parameters ${ }^{1}, X_{n_{0}^{\prime} n}$ represents the meansquare cross-spectral pressure per bandwidth, due to the coherent portion between the sources at $n_{0}^{\prime}$, and $n^{\prime}$, at each microphone $m$ (or $m^{\prime}$ ) normalized in level for a microphone at $r_{m}=r_{c}$. As will be seen, the determination of $X_{n_{0}^{\prime} n^{\prime}}$ is the primary objective of DAMAS-C.

Note, that if the sources at $n_{0}^{\prime}$, and $n^{\prime}$ each radiate noise in a statistically independent way then $X_{n_{0}^{\prime} n^{\prime}}=0$. In particular, if $X_{n_{0}^{\prime} n^{\prime}}=0$ when $n_{0}^{\prime} \neq n^{\prime}$, then the result from Ref. 1 is attained,

$$
G_{m m^{\prime}}=\sum_{n}^{N} X_{n n}\left(e_{m n}^{-1}\right)^{*} e_{m^{\prime} n}^{-1}
$$

where $G_{m m^{\prime}}$ are components of $\mathbf{G}_{\text {mod }}$ from Eq. (13) of Ref. 1. Here, $X_{n n}$ and $e_{m n}^{-1}$ equal $X_{n}$ and $e_{m: n}^{-1}$, respectively, of Ref. 1.

For our present coherent source case, the presently modeled CSM is $\mathbf{G}_{\bmod C}$ with components $G_{m m^{\prime}}$ given by Eq. (10). Employing this in Eq. (5),

$$
\left(Y_{n_{0} n}\right)_{\bmod }=\frac{\mathbf{e}_{n_{0}}{ }^{T} \mathbf{G}_{\bmod C} \mathbf{e}_{n}}{m_{0}^{2}}
$$




$$
\left(Y_{n_{0} n}\right)_{\bmod }=\frac{\mathbf{e}_{n_{0}}^{T} \sum_{n_{0}^{\prime}} \sum_{n^{\prime}} X_{n_{0}^{\prime} n^{\prime}}[]_{n_{0}^{\prime} n^{\prime}} \mathbf{e}_{n}}{m_{0}^{2}}=\frac{\sum_{n_{0}^{\prime}} \sum_{n^{\prime}}\left(\mathbf{e}_{n_{0}}^{T}[]_{n_{0}^{\prime} n^{\prime}} \mathbf{e}_{n}\right) X_{n_{0}^{\prime} n^{\prime}}}{m_{0}^{2}}
$$

where the bracketed term is

$$
[]_{n_{0}^{\prime} n^{\prime}}=\left[\begin{array}{cccc}
\left(e_{1 n_{0}^{\prime}}^{-1}\right)^{*} e_{1 n^{\prime}}^{-1} & \left(e_{1 n_{0}^{\prime}}^{-1}\right)^{*} e_{2 n^{\prime}}^{-1} & \cdots & \left(e_{1 n_{0}^{\prime}}^{-1}\right)^{*} e_{m_{0} n^{\prime}}^{-1} \\
\left(e_{2 n_{0}^{\prime}}^{-1}\right)^{*} e_{1 n^{\prime}}^{-1} & \left(e_{2 n_{0}^{\prime}}^{-1}\right)^{*} e_{2 n^{\prime}}^{-1} & & \vdots \\
\vdots & & \ddots & \vdots \\
\left(e_{m_{0} n_{0}^{\prime}}^{-1}\right)^{*} e_{1 n^{\prime}}^{-1} & \cdots & \cdots & \left(e_{m_{0} n_{0}^{\prime}}^{-1}\right)^{*} e_{m_{0} n^{\prime}}^{-1}
\end{array}\right]
$$

To be explicit, one can look at the terms of Eq. (13), with $n_{0}^{\prime}=1,2, \cdot, N$ and $n^{\prime}=1,2, \cdot, N$

$$
m_{0}^{2} Y_{n_{0} n}=\mathbf{e}_{n_{0}}^{T}[]_{11} \mathbf{e}_{n} X_{11}+\mathbf{e}_{n_{0}}^{T}[]_{12} \mathbf{e}_{n} X_{12}+\cdot+\mathbf{e}_{n_{0}}^{T}[]_{21} \mathbf{e}_{n} X_{21}+\cdots+\mathbf{e}_{n_{0}}^{T}[]_{N 1} \mathbf{e}_{n} X_{N 1}+\cdots+\mathbf{e}_{n_{0}}^{T}[]_{N N} \mathbf{e}_{n} X_{N N}
$$

And for $n_{0}=1,2, \cdot, N$ and $n=1,2, \cdot, N$

$$
\begin{aligned}
& m_{0}^{2} Y_{11}=\mathbf{e}_{1}^{T}[]_{11} \mathbf{e}_{1} X_{11}+\mathbf{e}_{1}^{T}[]_{12} \mathbf{e}_{1} X_{12}+\cdot \cdot+\mathbf{e}_{1}^{T}[]_{21} \mathbf{e}_{1} X_{21}+\cdot \cdot+\mathbf{e}_{1}^{T}[]_{N 1} \mathbf{e} X_{N 1}+\cdot \cdot+\mathbf{e}_{1}^{T}[]_{N N} \mathbf{e}_{1} X_{N N} \\
& m_{0}^{2} Y_{12}=\mathbf{e}_{1}^{T}[]_{11} \mathbf{e}_{2} X_{11}+\mathbf{e}_{1}^{T}[]_{12} \mathbf{e}_{2} X_{12}+\cdot \cdot+\mathbf{e}_{1}^{T}[]_{21} \mathbf{e}_{2} X_{21}+\cdot \cdot+\mathbf{e}_{1}^{T}[]_{N 1} \mathbf{e}_{2} X_{N 1}+\cdot \cdot+\mathbf{e}_{1}^{T}[]_{N N} \mathbf{e}_{2} X_{N N} \\
& m_{0}^{2} Y_{21}=\mathbf{e}_{2}^{T}[]_{11} \mathbf{e}_{1} X_{11}+\mathbf{e}_{2}^{T}[]_{12} \mathbf{e}_{1} X_{12}+\cdots+\mathbf{e}_{2}^{T}[]_{21} \mathbf{e}_{1} X_{21}+\cdot \cdot+\mathbf{e}_{2}^{T}[]_{N 1} \mathbf{e}_{1} X_{N 1}+\cdot+\mathbf{e}_{2}^{T}[]_{N N} \mathbf{e}_{1} X_{N N} \\
& m_{0}^{2} Y_{N 1}=\mathbf{e}_{N}^{T}[]_{11} \mathbf{e}_{1} X_{11}+\mathbf{e}_{N}^{T}[]_{12} \mathbf{e}_{1} X_{12}+\cdot \cdot+\mathbf{e}_{N}^{T}[]_{21} \mathbf{e}_{1} X_{21}+\cdot \cdot+\mathbf{e}_{N}^{T}[]_{N 1} \mathbf{e}_{1} X_{N 1}+\cdot+\mathbf{e}_{N}^{T}[]_{N N} \mathbf{e}_{1} X_{N N} \\
& m_{0}^{2} Y_{N N}=\mathbf{e}_{N}^{T}[]_{11} \mathbf{e}_{N} X_{11}+\mathbf{e}_{N}^{T}[]_{12} \mathbf{e}_{N} X_{12}+\cdots+\mathbf{e}_{N}^{T}[]_{21} \mathbf{e}_{N} X_{21}+\cdot \cdot+\mathbf{e}_{N}^{T}[]_{N 1} \mathbf{e}_{N} X_{N 1}+\cdots+\mathbf{e}_{N}^{T}[]_{N N} \mathbf{e}_{N} X_{N N}
\end{aligned}
$$

One notes that Eq. (16) can be written as

$$
\mathbf{Y}_{C}=\mathbf{A}_{C} \mathbf{X}_{C}
$$

that is the same inverse problem form as DAMAS, Eq. (18) of Ref. 1. Here, however, $\mathbf{Y}_{C}$ and $\mathbf{X}_{C}$ each have $N^{2}$ complex-number components, rather than the $N$ real-number components of Ref. 1 , that is

and

$$
\begin{gathered}
Y_{n_{0} n}, \text { with } n_{0} n=11,12, \cdot, 1 N, 21,22, \cdot \cdot, 2 N, 31,32, \cdot, N N \\
X_{n_{0} n}, \text { with } n_{0} n=11,12, \cdot \cdot 1 N, 21,22, \cdot \cdot 2 N, 31,32, \cdot, N N
\end{gathered}
$$

And $\mathbf{A}_{C}$ has $N^{4}$ complex-number components, rather than the previous $N^{2}$ real-number components, which are

$$
A_{n_{0} n, n_{0}^{\prime} n^{\prime}}=\left(\mathbf{e}_{n_{0}}^{T}[]_{n_{0}^{\prime} n^{\prime}} \mathbf{e}_{n}\right) / m_{0}^{2}
$$


with []$_{n_{0}^{\prime} n^{\prime}}$ being defined by Eq. (14) and the order within $\mathbf{A}_{C}$ defined by

$$
\mathbf{A}_{C}=\left[\begin{array}{cccccccc}
A_{11,11} & A_{11,12} & A_{11,13} & \cdots & A_{11,21} & A_{11,22} & \cdots & A_{11, N N} \\
A_{12,11} & A_{12,12} & A_{12,13} & & & & & \\
A_{13,11} & A_{13,12} & A_{13,13} & & & & & \\
& \vdots & & \ddots & & & & \vdots \\
A_{21,11} & A_{21,12} & & & \ddots & & & \\
A_{22,11} & A_{22,12} & & & & \ddots & & \\
& \vdots & & & & & A_{N N-1, N N-1} & A_{N N-1, N N} \\
A_{N N, 11} & A_{N N, 12} & & & \cdots & & A_{N N, N N-1} & A_{N N, N N}
\end{array}\right]
$$

The above equations contain terms that are complex conjugates of one another. These are

$$
Y_{n_{0} n}=Y_{n n_{0}}^{*}
$$

and

$$
X_{n_{0} n}=X_{n n_{0}}^{*}
$$

Relationships are also noted for $\mathbf{A}_{C}$. For the diagonal terms of Eq. (20), where one has $n_{0} n=n_{0}^{\prime} n^{\prime}$,

$$
A_{n_{0} n, n_{0}^{\prime} n^{\prime}}=A_{n_{0} n, n_{0} n}=1
$$

Also, for all terms, because of Eqs. (21) and (22), it can be shown that

$$
A_{n_{0} n, n_{0}^{\prime} n^{\prime}}=A_{n n_{0}, n^{\prime} n_{0}^{\prime}}^{*}
$$

whether $n_{0}=n$ or $n_{0} \neq n$ and whether $n_{0}^{\prime}=n^{\prime}$ or $n_{0}^{\prime} \neq n^{\prime}$. The terms are in general complex, but when $n_{0}=n$ and $n_{0}^{\prime}=n^{\prime}, A_{n_{0} n, n_{0}^{\prime} n^{\prime}}=A_{n_{0} n_{0}, n_{0}^{\prime} n_{0}^{\prime}}$ is real. (Note that the DAMAS problem of Ref. 1 is recovered if $X_{n_{0} n}=0$ when $n_{0} \neq n$ and one only considers the beamform power spectrum $Y_{n_{0} n_{0}}=Y_{n}$ rather than the cross-spectrum $Y_{n_{0} n}$. Then $A_{n_{0} n, n_{0}^{\prime} n^{\prime}}$ becomes $A_{n}$ of Ref. 1.)

The above relationships between terms, Eqs. (21)-(24), mean that the DAMAS-C problem contains $N(N+1) / 2$, rather than $N^{2}$, potentially independent equations and unknowns. In fact, the $Y_{n_{0} n}$ rows in Eq. (16) are complex conjugates of other $Y_{n_{0} n}$ rows. This means that for Eq. (17), the number of components can be reduced by almost half by removing rows. Then for $\mathbf{Y}_{C}=\mathbf{A}_{C} \mathbf{X}_{C}$, the components can be defined as $Y_{n_{0} n}, X_{n_{0}^{\prime} n^{\prime}}$, and $A_{n_{0} n, n_{0}^{\prime} n^{\prime}}$ with the indices $n_{0} n$ and $n_{0}^{\prime} n^{\prime}$ following the pattern,

$$
\begin{aligned}
n_{0} n & =11,12,13, \ldots, 1 N, 22,23,24, . ., 2 N, 33,34, \ldots, N N \\
& =\left(n_{0}=1,2,3, . ., N\right)\left(n=n_{0}, n_{0}+1, n_{0}+2, . ., N\right)
\end{aligned}
$$

and

$$
\begin{aligned}
n_{0}^{\prime} n^{\prime} & =11,12,13, \ldots, 1 N, 21,22,23, . ., 2 N, 31,32, . ., N N \\
& =\left(n_{0}^{\prime}=1,2,3, . ., N\right)\left(n^{\prime}=1,2,3, . ., N\right)
\end{aligned}
$$


Therefore, using Eqs, (25) and (26), the DAMAS-C problem, Eq. (17), is reduced in size from that indicated by Eqs. (18)-(20). $\quad \mathbf{Y}_{C}$ then has $N(N+1) / 2$ terms, $\mathbf{X}_{C}$ has $N^{2}$ terms $\left(N(N+1) / 2\right.$ are independent), and $\mathbf{A}_{C}$ has $N^{3}(N+1) / 2$ terms $\left(N^{2}\left(N^{2}+1\right) / 2\right.$ are independent).

Modified Beamforming. Note that the inverse problem, Eq. (17), for $\mathbf{X}_{C}$ can be defined in terms of $\mathbf{Y}_{C}$ and $\mathbf{A}_{C}$ being generated by shaded standard, diagonal removal (DR), and shaded DR beamforming - in parallel with that done in Ref. 1. That is, for shaded standard beamforming,

$$
Y_{n_{0} n}=\frac{\mathbf{e}_{n_{0}}^{T} \mathbf{W G W} \mathbf{W}^{T} \mathbf{e}_{n}}{\left(\sum_{m=1}^{m_{0}} w_{m}\right)^{2}}
$$

and, correspondingly, $\mathbf{A}_{C}$ is defined by components

$$
A_{n_{0} n, n_{0}^{\prime} n^{\prime}}=\frac{\mathbf{e}_{n_{0}}^{T} \mathbf{W}[]_{n_{0}^{\prime} n^{\prime}} \mathbf{W}^{T} \mathbf{e}_{n}}{\left(\sum_{m=1}^{m_{0}} w_{m}\right)^{2}}
$$

For diagonal removal, DR, beamforming

$$
Y_{n_{0} n}=\frac{\mathbf{e}_{n_{0}}^{T} \mathbf{G}_{\text {diag }=0} \mathbf{e}_{n}}{m_{0}^{2}-m_{0}}
$$

and, correspondingly, $\mathbf{A}_{C}$ is defined by components

$$
A_{n_{0} n, n_{0}^{\prime} n^{\prime}}=\frac{\mathbf{e}_{n_{0}}^{T}\left([]_{n_{0}^{\prime} n^{\prime}}\right)_{\operatorname{diag}=0} \mathbf{e}_{n}}{m_{0}{ }^{2}-m_{0}}
$$

For shaded DR beamforming

$$
Y_{n_{0} n}=\frac{\mathbf{e}_{n_{0}}^{T} \mathbf{W G}_{\text {diag }=0} \mathbf{W}^{T} \mathbf{e}_{n}}{\left(\sum_{m=1}^{m_{0}} w_{m}\right)^{2}-\left(\sum_{m=1}^{m_{0}} w_{m}\right)}
$$

and, correspondingly, $\mathbf{A}_{C}$ is defined by components

$$
A_{n_{0} n, n_{0}^{\prime} n^{\prime}}=\frac{\mathbf{e}_{n_{0}}^{T} \mathbf{W}\left([]_{n_{0}^{\prime} n^{\prime}}\right)_{\operatorname{diag}=0} \mathbf{W}^{T} \mathbf{e}_{n}}{\left(\sum_{m=1}^{m_{0}} w_{m}\right)^{2}-\left(\sum_{m=1}^{m_{0}} w_{m}\right)}
$$

Note that for all the above types of special beamforming processing, the relationships Eqs. (21)-(26) are equally valid. 
DAMAS-C Inverse Problem Solution. As in Eq. (22) of Ref. 1, a single linear equation component of the present Eq. (17) is

$$
\begin{gathered}
Y_{n_{0} n}=A_{n_{0} n, 11} X_{11}+A_{n_{0} n, 12} X_{12}+\cdot \cdot+A_{n_{0} n, 1 N} X_{1 N}+A_{n_{0} n, 21} X_{21}+A_{n_{0} n, 22} X_{22}+\cdot \cdot+A_{n_{0} n, N N} X_{N N} \\
Y_{n_{0} n}=\sum_{n_{0}^{\prime}=1}^{N} \sum_{n^{\prime}=1}^{N} A_{n_{0} n, n_{0}^{\prime} n^{\prime}} X_{n_{0}^{\prime} n^{\prime}}
\end{gathered}
$$

With $A_{n_{0} n, n_{0} n}=1$ from Eq. (23), this is rearranged to give

$$
X_{n_{0} n}=Y_{n_{0} n}-\left[\sum_{n_{0}^{\prime}=1}^{n_{0}} \sum_{n^{\prime}=1}^{n-1} A_{n_{0} n, n_{0}^{\prime} n^{\prime}} X_{n_{0}^{\prime} n^{\prime}}+\sum_{n_{0}^{\prime}=n_{0}}^{N} \sum_{n^{\prime}=n+1}^{N} A_{n_{0} n, n_{0}^{\prime} n^{\prime}} X_{n_{0}^{\prime} n^{\prime}}\right]
$$

Because of the counting pattern of $n_{0}^{\prime} n^{\prime}$ from Eq. (26), exceptions are noted in the upper and lower sum limits in Eq. (35). If $X_{n_{0} n}=X_{n_{0} 1}$, then the upper limits on the first set of sums, i.e. $n_{0}$ and $n-1$, are replaced by $n_{0}-1$ and $N$, respectively, and the lower limits on the second set of sums remain as they are. But, if $X_{n_{0} n}=X_{n_{0} N}$, then although the upper limits on the first set of sums remain as they are, the lower limits on the second set of sums, i.e. $n_{0}$ and $n+1$ are replaced by $n_{0}+1$ and 1 .

Equation (35) is used in an iteration algorithm to obtain the source distribution strengths $X_{n n}$ (or $X_{n_{0} n_{0}}$ ) for all $n$ and cross-strengths $X_{n_{0} n}$ for all combinations of $n_{0}$ and $n$ indicated by Eq. (25), as per the following equation.

$$
\begin{gathered}
X_{11}^{(i)}=Y_{11}-\left[0+\sum_{n_{0}^{\prime}=1}^{N} \sum_{n^{\prime}=1+1}^{N} A_{11, n_{0}^{\prime} n^{\prime}} X_{n_{0}^{\prime} n^{\prime}}^{(i-1)}\right] \\
X_{n_{0} n}^{(i)}=Y_{n_{0} n}-\left[\sum_{n_{0}^{\prime}=1}^{n_{0}} \sum_{n^{\prime}=1}^{n-1} A_{n_{0} n, n_{0}^{\prime} n^{\prime}} X_{n_{0}^{\prime} n^{\prime}}^{(i)}+\sum_{n_{0}^{\prime}=n_{0}}^{N} \sum_{n^{\prime}=n+1}^{N} A_{n_{0} n, n_{0}^{\prime} n^{\prime}} X_{n_{0}^{\prime} n^{\prime}}^{(i-1)}\right] \\
X_{N N}^{(i)}=Y_{N N}-\left[\sum_{n_{0}^{\prime}=1}^{N} \sum_{n^{\prime}=1}^{N-1} A_{N N, n_{0}^{\prime} n^{\prime}} X_{n_{0}^{\prime} n^{\prime}}^{(i)}+0\right]
\end{gathered}
$$

Here, the first and last term of the $i^{\text {th }}$ iteration, (i), for $X_{n_{0} n}$ is shown for clarity. Equation (36) represents a similar form as that given in Eq. (24) of Ref. 1, except that these terms are complex and that the problem size for the same number of $N$ grid points is expanded. In the iterations, the same sum limit exceptions mentioned for Eq. (35) apply.

The iteration path is consistent with a progression through a stack of $n_{0}=1,2,3, \ldots, N$ solution maps (planes). Figure 3 illustrates the $N$ planes, where within each, the counting sequence starts at $n=1$ in the left bottom corner and increases vertically along each column. The uppermost point in the last column to the right is the $n=N$ grid point. The last three frames (planes) of Fig. 2 show $Y_{n_{0} n}$ magnitude levels for the particular $n_{0}$ planes shown. (The $Y_{n_{0} n_{0}}$ map of Fig. 2 is not a $n_{0}$ plane, but one made up of points $Y_{n_{0} n_{0}}$ from each $n_{0}$ plane.) For $Y_{n_{0} n}$ terms, as well as for $X_{n_{0} n}$, with only $N+1-n_{0}$ terms calculated for each $n_{0}$ plane, the values in the plots for the remaining $n_{0}-1$ grid points are determined from the complex conjugate relationships. The $Y_{n_{0} n}$ terms are defined from Eqs. (5), (27), (29), or (31). The $A_{n_{0} n, n_{0}^{\prime} n^{\prime}}$ terms are determined from Eq. (19), (28), (30), or (32). For each (i) iteration for the $X_{n_{0} n}$ terms, the values of $X_{n_{0} n}^{(i)}$ replace the previous iteration $X_{n_{0} n}^{(i-1)}$ values. Therefore, whereas Eq. (17) with the 
appropriate $A_{C}$ defines the DAMAS-C inverse problem, Eq. (36) is the inverse problem iterative solution, with the appropriate constraints described below. ${ }^{10}$

Physical Interpretations and Positivity Constraints. In Ref. 1, a positivity constraint was used in the iterations for $X_{n}$. This made the inverse DAMAS problem for $X_{n}$ sufficiently deterministic to force unique solutions. The constraint is actually physically necessary in that $X_{n}$ represents auto-spectral pressure-squared (positive) amplitude for the sources at grid points $n$. In DAMAS-C, the value of $X_{n_{0} n}$ is $\operatorname{Re}\left(X_{n_{0} n}\right)+\operatorname{Im}\left(X_{n_{0} n}\right)$. When $n=n_{0}, X_{n_{0} n}=X_{n_{0} n_{0}}$ is real and positive - equivalent to the DAMAS $X_{n}$. Thus for each iteration calculation for Eq. (36), $\operatorname{Im}\left(X_{n_{0} n_{0}}\right)$ is set to zero and $\operatorname{Re}\left(X_{n_{0} n_{0}}\right)$ is set to zero if it is not already positive. This is equivalent to the positivity constraint $X_{n}$ of Ref. 1 .

When $n_{0} \neq n, X_{n_{0} n}$ could be in any of four complex quadrants. In terms of a complex coherence definition

$$
X_{n_{0} n}=\gamma_{n_{0} n} \sqrt{X_{n n}} \sqrt{X_{n_{0} n_{0}}}
$$

where the coherence

$$
\begin{gathered}
\overline{\gamma_{n_{0} n}^{2}}=\overline{\gamma_{n n_{0}}^{2}}=\overline{X_{n_{0} n}^{2}} / X_{n_{0} n_{0}} X_{n n}=X_{n_{0} n}^{*} X_{n_{0} n} / X_{n_{0} n_{0}} X_{n n} \\
\gamma_{n_{0} n}=\sqrt{\overline{\gamma_{n_{0} n}^{2}}} \exp \left(i \varphi_{n_{0} n}\right)
\end{gathered}
$$

where $\varphi_{n_{0} n}$ is the cross-spectral phase or phase between coherent portions of source strength at point $n$ with respect to $n_{0}$. This of course follows from $X_{n_{0} n}=X_{n n_{0}}^{*}$. Physically, $\gamma_{n_{0} n}$ is interpreted here as the coherence factor between the sources at $n$ and $n_{0}$. It can be related to noise emission from unsteady aerodynamic related regions over radiating surfaces $\left(0 \leq\left|\gamma_{n_{0} n}\right| \leq 1\right.$, for $n_{0}$ and $n$ being nearby radiating locations) or reflections, such as from image regions in the presence of tunnel sidewalls, $\left(\left|\gamma_{n_{0} n}\right| \approx 1\right.$ for $n_{0}$ being the source and $n$ being the reflection image).

An appropriate constraint based on the above equations is that $\left|X_{n_{0} n}\right| \leq \sqrt{X_{n_{0} n_{0}} X_{n n}}$ be enforced in the iterations. This is not done in the coding for the present paper. Instead, $X_{n_{0} n}$ is regarded as an independent variable in the same manner as $X_{n_{0} n_{0}}$ and $X_{n n}$. Also, with regard to the generality of $X_{n_{0} n}$, there is a question concerning the rank of the DAMAS-C inverse problem, and thus the practicality of solving for $X_{n_{0} n}$ with arbitrary phase. If the inverse problem, Eq. (17), is of low rank (more unknowns than independent equations) this would mean that the problem is indeterminate for a unrestricted solution space. That would suggest that the solution space should be limited, such that was used for DAMAS with its positivity constraint. For the present paper, DAMAS-C applications are limited to sources that have only in-phase coherence, that is $\varphi_{n_{0} n}=0$ in Eq. (39), as a constraint. In this $n_{0} \neq n$ case, after each iteration calculation (as is done above for $\left.n_{0}=n\right), \operatorname{Im}\left(X_{n_{0} n}\right)$ is set to zero and $\operatorname{Re}\left(X_{n_{0} n}\right)$ is set to zero if it is not already positive.

Problem Reduction by Zoning. The size of the matrices of $\mathbf{Y}_{C}=\mathbf{A}_{C} \mathbf{X}_{C}$ can be quite large and prohibitive for many evaluation regions required in practical DAMAS-C applications. The storage of these large numbers of terms, combined with the iteration procedure requirements, require the use of methods that can further reduce the problem size wherever the physical problem permits. A method was developed called zoning, which is employed to restrict the possible solutions to anticipated or realizable conditions of the noise source evaluation region under study. The evaluation region can be composed of a number of grid point zones, each with assumed coherence criteria. The criteria can be uniform over the zones or functionally dependent on, for example, the point-to-point distance and frequency.

For the present paper, the source evaluation region (which can be contiguous or not) is composed of multiple non-congruent zones $\mathrm{A}$ and $\mathrm{B}$ containing grid points $(n)_{\mathrm{A}}$ and $(n)_{\mathrm{B}}$, respectively. The zone $\mathrm{A}$ is taken as a region 
of coherent sources. Zone B is composed completely of incoherent sources. This means that cross terms $X_{\left(n_{0}^{\prime}\right)_{\mathrm{A}}\left(n^{\prime}\right)_{\mathrm{B}}}$ are zero. $X_{\left(n_{0}^{\prime}\right)_{\mathrm{B}}\left(n^{\prime}\right)_{\mathrm{B}}}$ is zero when $n_{0} \neq n$. This zeros out the corresponding $\mathbf{A}_{C}$ matrix columns. To maintain the iteration scheme of Eq. (36), the cross $\mathbf{Y}_{C}$ terms corresponding to the cross $\mathbf{X}_{C}$ terms, identified above, and the corresponding $\mathbf{A}_{C}$ matrix rows are eliminated. Although these cross $\mathbf{Y}_{C}$ terms are not zero, their elimination reduces the number of unknowns and corresponding equations while still giving weight in the solutions to a broader region (zone B) through the auto terms of $\mathbf{Y}_{C}$. The reduction in problem size can be substantial. Zone A has $N_{\mathrm{A}}$ grid points, zone $\mathrm{B}$ has $N_{\mathrm{B}}$, and the total is $N=N_{\mathrm{A}}+N_{\mathrm{B}}$. The number of independent $\mathbf{X}_{C}$ and $\mathbf{Y}_{C}$ terms are $\left[N_{\mathrm{A}}\left(N_{\mathrm{A}}+1\right) / 2+N_{\mathrm{B}}\right]$ and the number of $\mathbf{A}_{C}$ terms is $\left[N_{\mathrm{A}}^{2}+N_{\mathrm{B}}\right]$ times that. For an example of the size problem, in regular DAMAS, a 33x33 grid point region would render 1089 grid points with a corresponding number of $\mathbf{X}$ and $\mathbf{Y}$ terms. Then A would have $(1089)^{2}=1.19 \times 10^{6}$ elements. But for DAMAS-C, the same grid region considered all in a zone $A$ would render a very large $5.94 \times 10^{5}$ for independent $\mathbf{X}_{C}$ and $\mathbf{Y}_{C}$ terms, and $7.04 \times 10^{11} \mathbf{A}_{C}$ terms. If, however, zone $A$ were made a $15 \times 15$ grid point region, DAMAS-C would have $2.54 \times 10^{4}$ independent $\mathbf{X}_{C}$ and $\mathbf{Y}_{C}$ terms and $1.29 \times 10^{9} \quad \mathbf{A}_{C}$ terms. Adding a $33 \times 33-15 \times 15=864$ point region in a zone $\mathrm{B}$ would increase these terms by $4 \%$ and $5 \%$, respectively. These are still very large numbers, but they pale in comparison to that found using a fully coherent $33 \times 33$ grid point zone $A$.

Computational Notes for the Present Paper. With respect to processing for this paper, it was found impractical to implement DAMAS-C on a single processor system. Therefore, a partial parallel implementation was performed on a 32-node Beowulf distributed memory computer. Only a partial parallel implementation could be achieved due to the nature of the DAMAS-C iterative solution. The DAMAS-C algorithm, Eq. (36), incorporates a relaxation method for solving the linear system, where the solver requires immediate access to all previously computed $\mathbf{X}_{C}$ terms during each iteration step. As such, the technique is serial in nature and cannot easily be used in shared memory parallel computing. Thus, a hybrid approach was taken, where the generation of the $\mathbf{A}_{C}$ matrix was implemented in parallel, but where the generation of the $\mathbf{Y}_{C}$ matrix and the running of the iterative solver were performed serially on one or more compute nodes.

An exception is noted for the algorithm implementation description for Eq. (36) for the calculations of this paper. For interim coding simplicity, Eq. (26) was used rather than Eq. (25) for the index definitions of $n_{0} n$, thereby keeping the number of $\mathbf{Y}_{C}$ and $\mathbf{X}_{C}$ terms at $N^{2}$ and those of $\mathbf{A}_{C}$ terms at $N^{4}$ in zone A. Although this is a larger problem than that described in the above discussions, this disadvantage was offset in part by employing the complex conjugate relationships Eqs. (21)-(24) to increase iterative efficiency.

\section{APPLICATIONS}

Simulated Sources. To illustrate the methodology, DAMAS-C is first applied to the simulated point source case of Fig. 2. This is the simplest of cases, without any issue of coherence or multiple sources. The example is of a scan plane placed $60 \mathrm{in}$. away from the $7.8 \mathrm{in}$. diameter SADA microphone array ${ }^{1}$. The frequency is $f=20 \mathrm{kHz}$. The scan plane is comprised of a $15 \times 15$ grid pattern of points $\Delta x=\Delta y=1.5 \mathrm{in}$. apart, so the height $H$ and width $W$ are 21 in. each. The half-power ( $3 \mathrm{~dB}$-down) beamwidth, designated $B_{\text {auto }}$ for the standard beamform processing for $Y_{n_{0} n_{0}}$ (Eq. (1)) is indicated in Fig. 2 to be approximately 7 in. The corresponding beamwidth $B_{\text {cross }}$ for $Y_{n_{0} n}$ in Fig. 2 is approximately 10.5 inches. (Note that the ratio of $B_{\text {cross }} / B_{\text {auto }}(10.5 / 7$ here $)$ depends on beam characteristics, but would have a maximum limiting value of 2 .) The criteria given in Ref. 1 for a resolution range that is considered reasonable for use in DAMAS are presumed to be applicable here. The criteria are

$$
0.05 \leq \Delta x / B(\text { or } \Delta y / B) \leq 0.2
$$

and

$$
1 \leq W / B(\text { and } H / B)
$$

This is approximately met here with $\Delta x / B_{\text {auto }}=.21, W / B_{\text {auto }}=3.0, \Delta x / B_{\text {cross }}=.14$, and $W / B_{\text {cross }}=2.0$. 


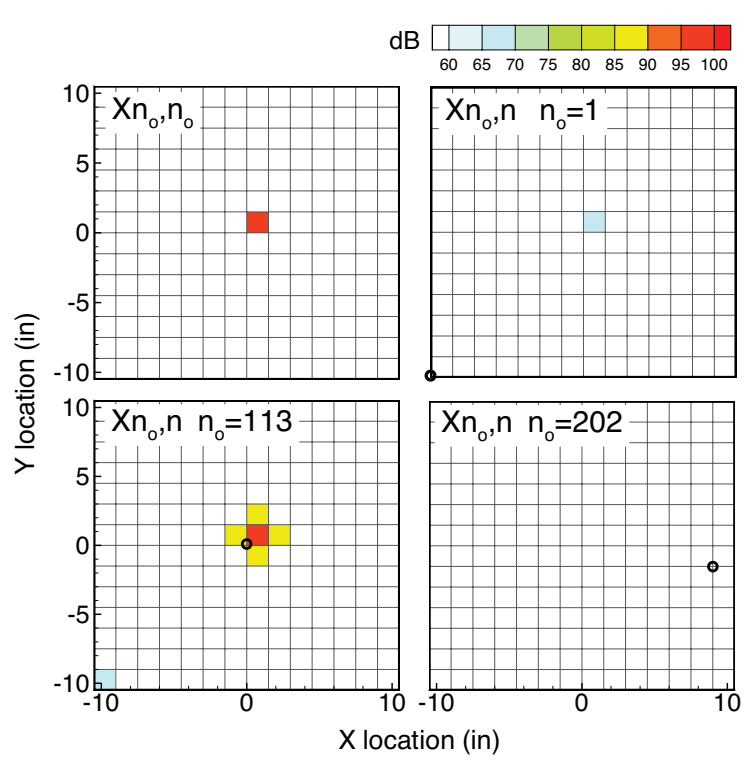

Figure 4. DAMAS-C results of source strengths $X_{n_{0} n_{0}}$ and cross strengths $X_{n_{0} n}$ between grid points at $n_{0}$ and $n$ over scan planes. Corresponds to Fig. 2.
All 225 grid points are considered in zone $A$ where coherence between points is permitted. For Fig. 2, the synthetic point source is positioned at $n=113$. This is done by defining $X_{113113}$ to give $100 \mathrm{~dB}=10 \log X_{113113}$ and all other $X_{n_{0}^{\prime} n^{\prime}}$ terms are set to zero in Eq. (13). Then $Y_{n_{0} n}$ is solved for over the scan plane and plotted in Fig. 2. Figure 4 shows the DAMAS-C results for $X_{n_{0} n_{0}}$ and $X_{n_{0} n}$, corresponding to the $Y_{n_{0} n_{0}}$ and $Y_{n_{0} n}$ plots of Fig. 2. These were determined from the algorithm of Eq. (37) using $i=100$ iterations. The iterations were started using $X_{n_{0} n}$ set to zero (little improvement is found when starting differently). Figure 4 show $X_{n_{0} n_{0}}$ levels, which represent the collection of $X_{n_{0} n}$ values, when $n=n_{0}$, from the individual $n_{0}$ planes. In the $n_{0}=1,113$, and 202 planes, the $n_{0}$ number locations are indicated by the open symbol, as done in Fig. 2. The levels are defined by color code in "blocks" (defined by the space between grid points) above and to the right of the grid point. It is seen that DAMAS$\mathrm{C}$, with this number of iterations, approaches the correct source definition. The value recovered for $X_{113113}$ itself is $96.5 \mathrm{~dB}$ (exact answer is $100 \mathrm{~dB}$ ), and the nearby $X_{n_{0} n}$ levels are about $89 \mathrm{~dB}$ (exact answer is $-\infty \mathrm{dB}$ ), and the total of all grid points is $100.2 \mathrm{~dB}$ (exact answer is 100 $\mathrm{dB})$. The number of iterations, $i$, chosen here is arbitrary. An increase in $i$ would increase accuracy, but is stopped at 100 to illustrate "energy" smearing due to limited iteration number. (Smearing also occurs with relation to too small $\Delta x / B$ and $W / B$ resolution values ${ }^{1}$, defined in Eqs. (43) and (44).)

To test the ability of DAMAS-C to separate and quantify sources, one can consider two point sources located 9 in. apart. Again the SADA is 5 feet away and the frequency is $f=20 \mathrm{kHz}$. First, Fig. 5 shows results for the two sources that are incoherent with respect to one another. The sources are positioned on a $51 \times 51$ inch scan plane with a grid point spacing of $\Delta x=\Delta y=1 \mathrm{in}$. With this spacing, $\Delta x / B_{\text {auto }}=.14$ and $\Delta x / B_{\text {cross }}=.10$. The top left frame shows a Diagonal Removal (DR) beamform processing (Eq. (29)) result contour plotted for $Y_{n_{0} n_{0}}$. (The dashed contour regions are actually regions of negative "pressure squared" values ${ }^{1,6}$, which occur because of the DR processing. For plotting purposes, the corresponding $\mathrm{dB}$ levels shown are defined using absolute values of the negative terms. A negative sign is simply applied to these levels.) The top right frame of Fig. 5 is a DAMAS processed result using the method of Ref. 1 for DR processing. Here, with DAMAS being perfectly applicable with the present incoherent sources, the sources are correctly located and levels of $100 \mathrm{~dB}$ for each are found. The level corresponding to the sum over all grid points in the frame is given (equated to the $\Sigma p^{2}$ symbol) as 103.0 dB. Results are given for DAMAS-C, where a zone A (with 153 points) and a zone B (with 2448 points) combination is used. The number of iterations is 200, rather than the 2000 employed for DAMAS ${ }^{1}$, because of the more significant computational requirements for DAMAS-C. The results shown in successive frames are the distributions of $X_{n_{0} n_{0}}, \Sigma X_{n_{0} n}$ (sum over all $n_{0}$ planes), $X_{n_{0} n}$ (for source location $n_{0}=1097$ plane), and $X_{n_{0} n}$ (for source location $n_{0}=1556$ plane). It is seen that DAMAS-C correctly identifies the sources. The summed level over the $X_{n_{0} n_{0}}$ frame of $97.2 \mathrm{~dB}$ (ideally $103 \mathrm{~dB}$ ) is the result of "energy" smearing due to the limited iterations and the many more $n_{0}$ planes that "energy" can spread. The summed level over the $\Sigma X_{n_{0} n}$ frame of $103.3 \mathrm{~dB}$ correctly matches the original DAMAS result of $103 \mathrm{~dB}$ showing that "energy" is conserved between DAMAS and DAMAS-C. The last two frames show the key result that DAMAS-C correctly separates the sources and validates that the two sources have no coherence with one another. 


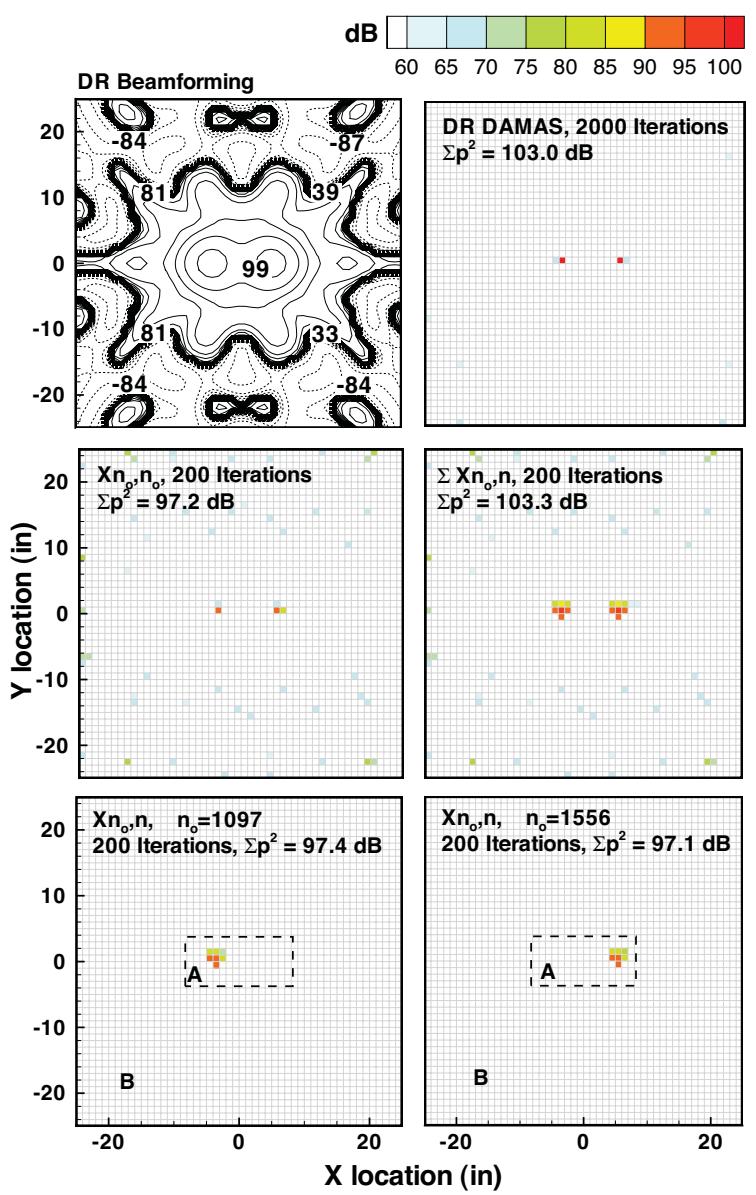

Figure 5. Beamforming (top left frame), DAMAS (top right frame), and DAMAS-C (last 4 frames) results for INCOHERENT point sources, 9 in. apart. $f=20 \mathrm{kHz}$.
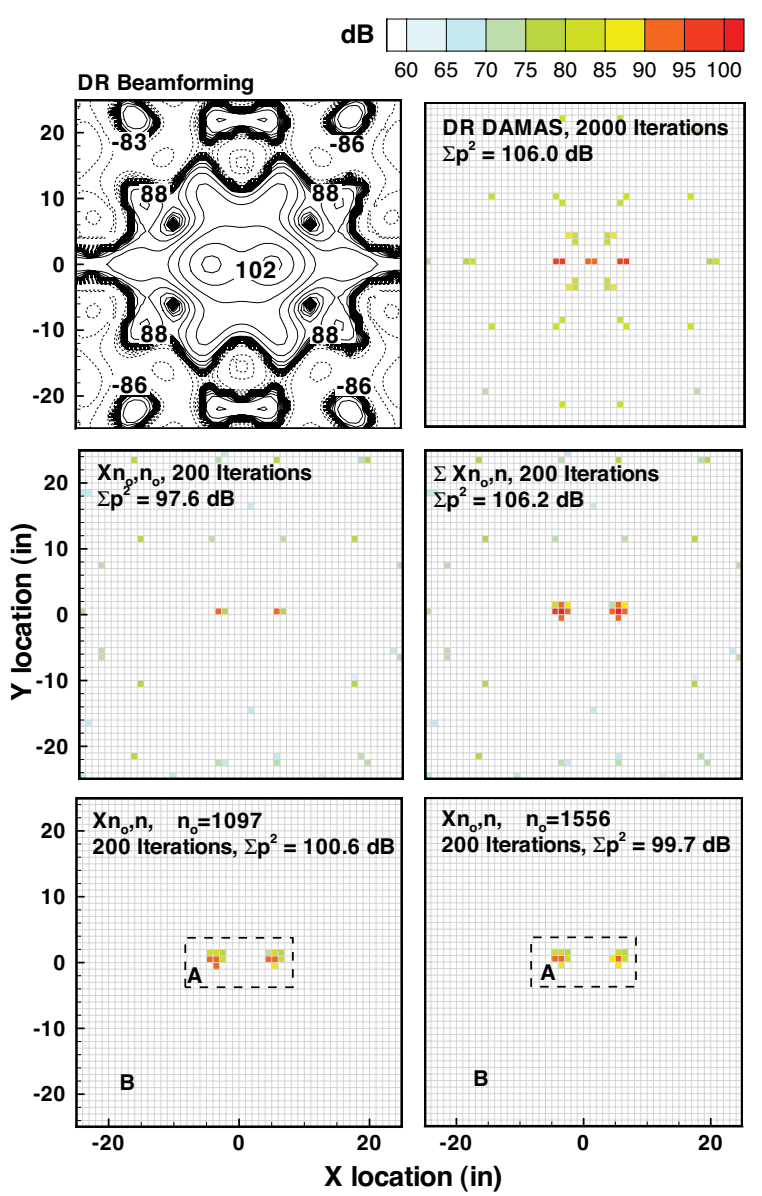

Figure 6. Same key as Fig. 5 except for COHERENT sources, 9 in. apart.

Figure 6 shows results for the two sources that are defined as perfectly coherent and in-phase. Figure 6 is identical in format to that of Fig. 5. It is seen that the beamform processing for $Y_{n_{0} n_{0}}$ is significantly affected by the coherence of the sources (compare this to Fig. 5). The corresponding original DAMAS processing of Fig. 6 is seen to produce a geometrically distorted result, although the sum of the apparent sources do correctly sum to $106 \mathrm{~dB}$. DAMAS-C is seen to correctly separate and quantify the coherent sources. The $\Sigma X_{n_{0} n}$ frame of 106.2 dB correctly matches level. (To be clear, with each of the coherent $X_{n_{0} n_{0}}, X_{n n}, X_{n_{0} n}$, and $X_{n n_{0}}$ source strengths having levels of $100 \mathrm{~dB}$, two should total $103 \mathrm{~dB}$, and all four should total $106 \mathrm{~dB}$.) However, the summed levels over $X_{n_{0} n_{0}}, X_{n_{0} n}$ ( $\left.n_{0}=1097\right)$, and $X_{n_{0} n}\left(n_{0}=1556\right)$ planes of 97.6, 100.6, and $99.7 \mathrm{~dB}$ are all lower than the ideal $103 \mathrm{~dB}$ level. Still, this is completely explained by the same resolution reasons given with respect to Fig. 5. Therefore, Figs. (5) and (6) validates the correctness and functionality of the DAMAS-C algorithm.

Figures 7 and 8 show a similar set of presentations to that of Figs. 5 and 6 , except that the sources were placed 4.5 inches apart rather than $9 \mathrm{in}$. In these simulations, $\Delta x=\Delta y=.9$ inches and frequency is again $20 \mathrm{kHz}$. It is seen for the incoherent sources, in Fig. 7, that almost the same degree of success in source definition is found using DAMAS-C for this closer source position. However, somewhat more difficulty is found when the sources are coherent. In Fig. 8, the totaled $\Sigma X_{n_{0} n}$ frame result appears almost as a line and the $X_{n_{0} n}$ frames shows smearing between the sources. In Ref. 1, there is defined a spatial detail dimension $\ell$ that was judged to be well resolvable by DAMAS if $2<\ell / B$, acceptably resolvable if $1 \leq \ell / B$, but only marginally for $\ell / B<1$. Here, upon associating $\ell$ 


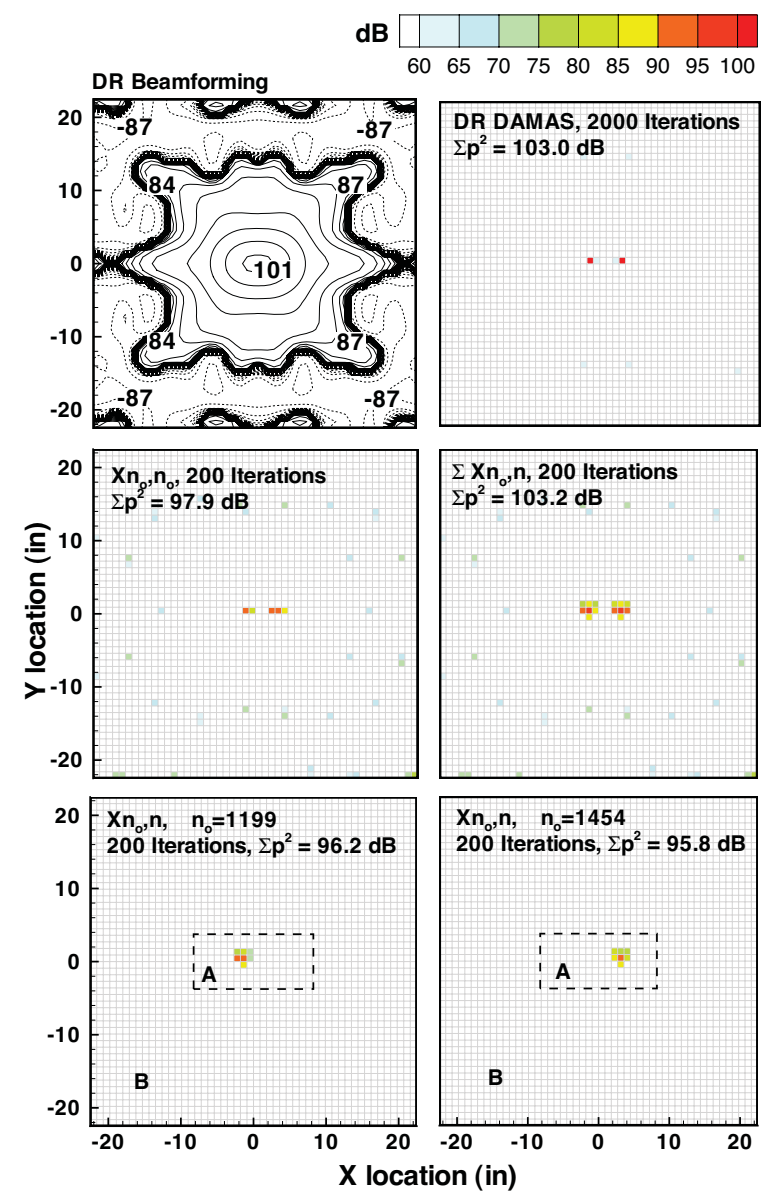

Figure 7. . Beamforming (top left frame), DAMAS (top right frame), and DAMAS-C (last 4 frames) results for for INCOHERENT point sources, 4.5 in. apart. $f=20 \mathrm{kHz}$.

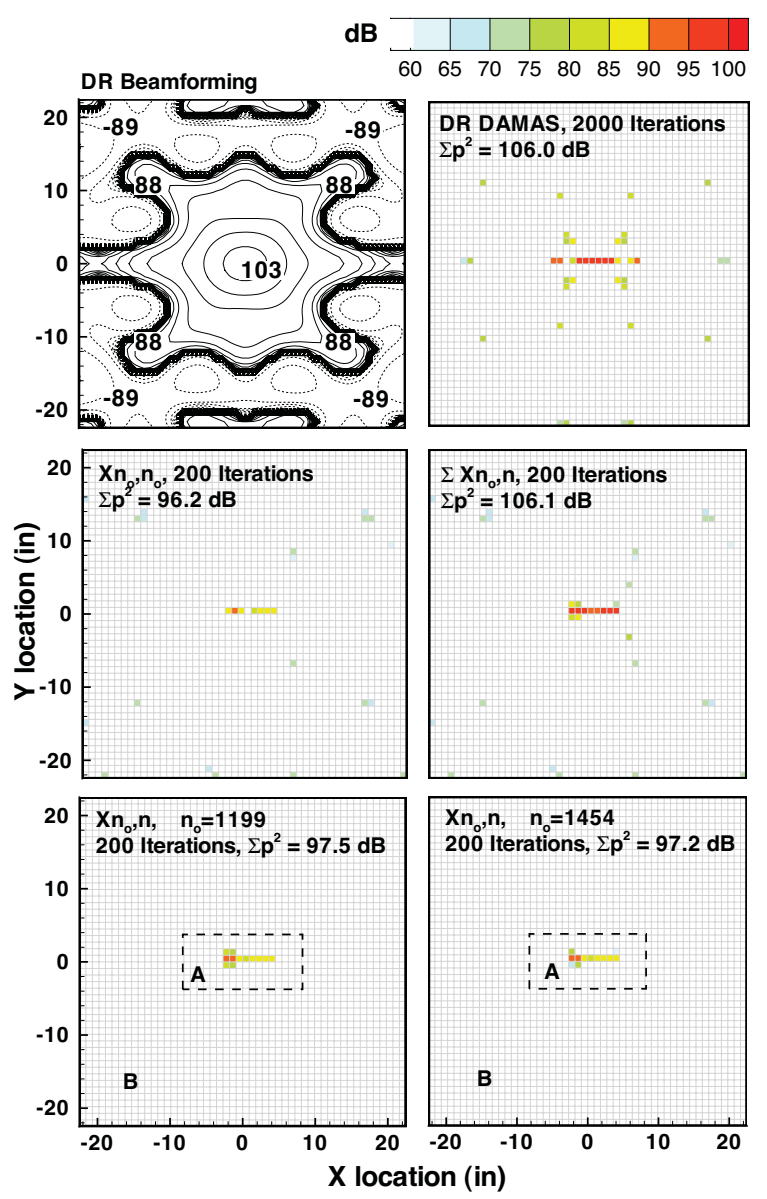

Figure 8. Same key as Fig. 7 except for COHERENT sources, 4.5 in. apart.

with the 4.5 in. separation, $\ell / B_{\text {auto }}=.6$ and $\ell / B_{\text {cross }}=.4$, which suggests marginal resolution. The present DAMAS$\mathrm{C}$ results then appear compatible with these criteria.

Figures 9 and 10 show a set of presentations, similar to the preceding, except that thirteen sources are distributed to simulate a 12-inch line source. Figure 9 shows for the incoherent line source that both DAMAS and DAMAS-C gives good spatial and level definition. The sums total about $111 \mathrm{~dB}$ (in agreement with $100+$ $10 \log (13) \mathrm{dB}$ ). The individual $n_{0}$ planes sum to the ideal $100 \mathrm{~dB}$ (aided partly in its summing by the observed lateral "energy" smearing about the respective $n_{0}$ locations). Figure 10 , for coherent line source, shows a substantially distorted result for DAMAS, although it is again shown that the total levels for both DAMAS and DAMAS-C are both correct at $122 \mathrm{~dB}\left(100+10 \log \left(13^{2}\right) \mathrm{dB}\right)$. The levels for the $n_{0}$ planes are similarly correct.

Experimental Data. DAMAS-C is applied to data from an airframe noise test in the Quiet Flow Facility (QFF) at NASA Langley Research Center. In Fig. 11, the flap edge test ${ }^{6,10}$ configuration is shown where the SADA array is positioned outside the flow field, at a distance of 5 feet from the model. The test case considered is for a $29^{\circ}$ flap angle setting and $M=0.11$. For this case, Ref. 1 and 3 showed that there was strong localized flap edge noise and distributed noise over the flap cove region. The sources are evaluated along a scanning plane that is aligned with the airfoil main element chordline. The DR beamform processing and corresponding DAMAS results are shown in Fig. 12. The presentation is for a $20 \mathrm{kHz}$ one-third octave frequency band, obtained by combining CSM results from a number of individual 17. $44 \mathrm{~Hz}$ bands to obtain $\Delta f=122 \mathrm{~Hz}$, used for evaluations of beamforming, DAMAS, and 


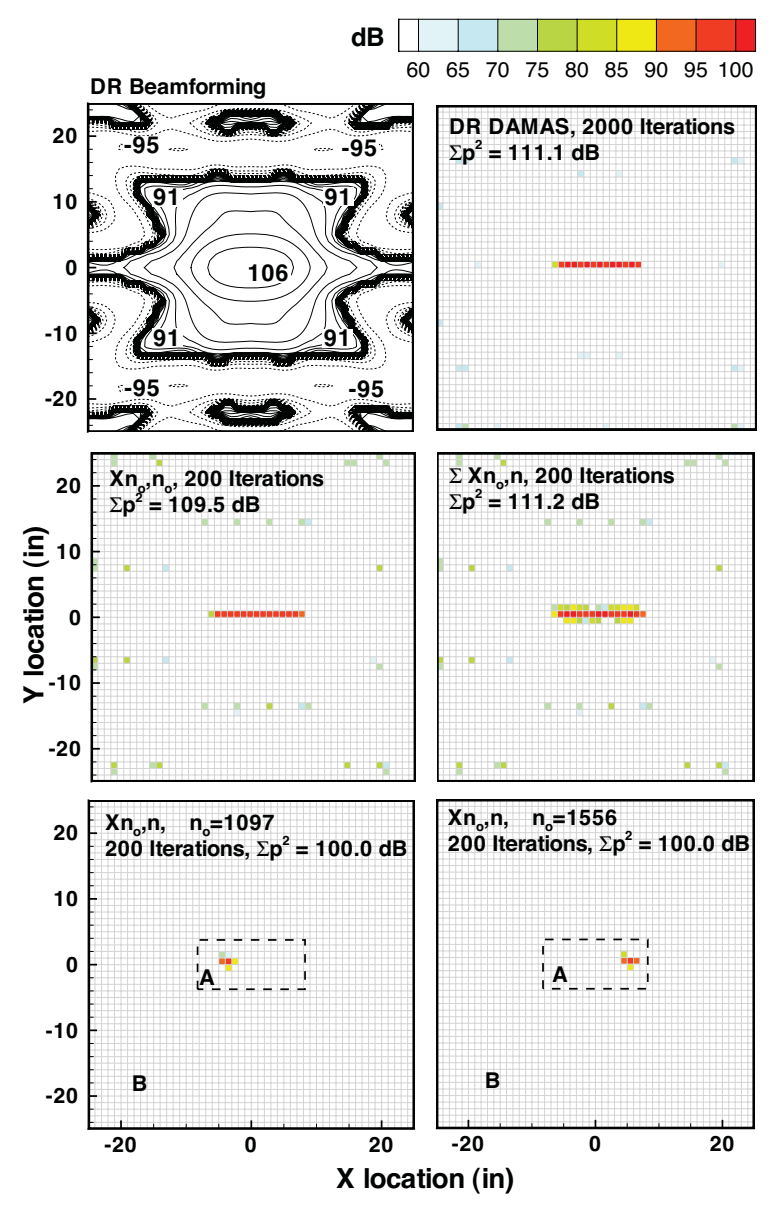

Figure 9. . Beamforming (top left frame), DAMAS (top right frame), and DAMAS-C (last 4 frames) results for for INCOHERENT line source. Frequency $f=20 \mathrm{kHz}$.

DAMAS-C. The individual results are then summed to obtain the one-third octave results shown. The zone A is a $9 \times 24$ point region over the scan plane as shown. Grid spacing is $\Delta x=\Delta y=1 \mathrm{in}$.

The results for the DAMAS and the totaled $\Sigma X_{n_{0} n}$ frame result of DAMAS-C substantially match in source distribution and level. This and the very consistent results for the $X_{n_{0} n_{0}}$ and $X_{n_{0} n}$ in the $n_{0}$ planes indicate that the flap edge and flap cove noise regions can be regarded as distributions of incoherent sources - at least to the extent that is resolvable for this size array and processing. However, alternately, one could view the apparent spatial coherence regions about the $n_{0}$ locations in the respective $n_{0}$ planes as coherent length scales of 3 to 4 inches. Countering this, however, is that these regions are also consistent with spatial "energy" smearing experienced in the foregoing simulations due to resolution and iteration limitations.

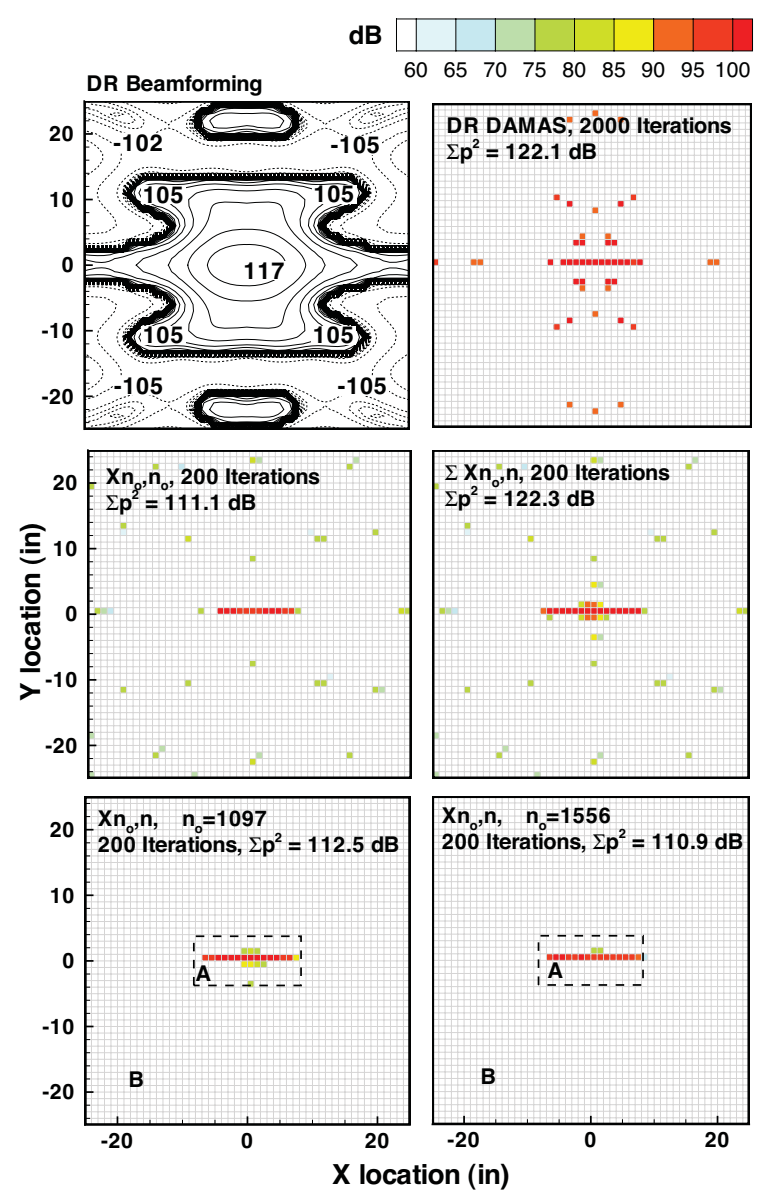

Figure 10. Same key as Fig. 9 except for COHERENT line source.

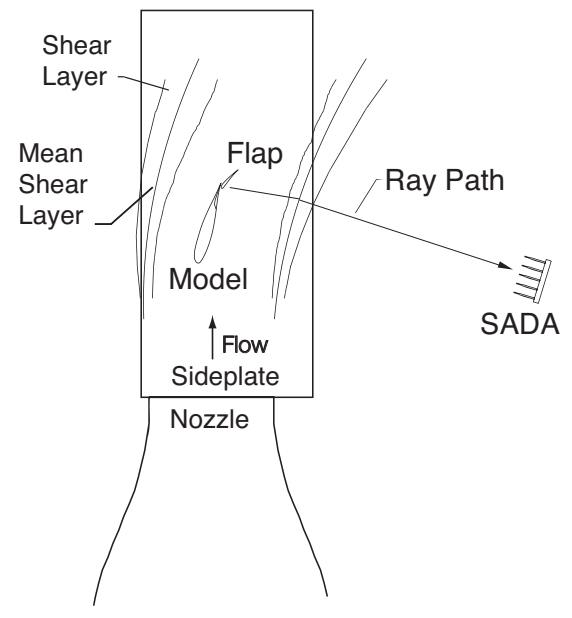

Figure 11. Sketch of flap noise test setup in the QFF open test section. 


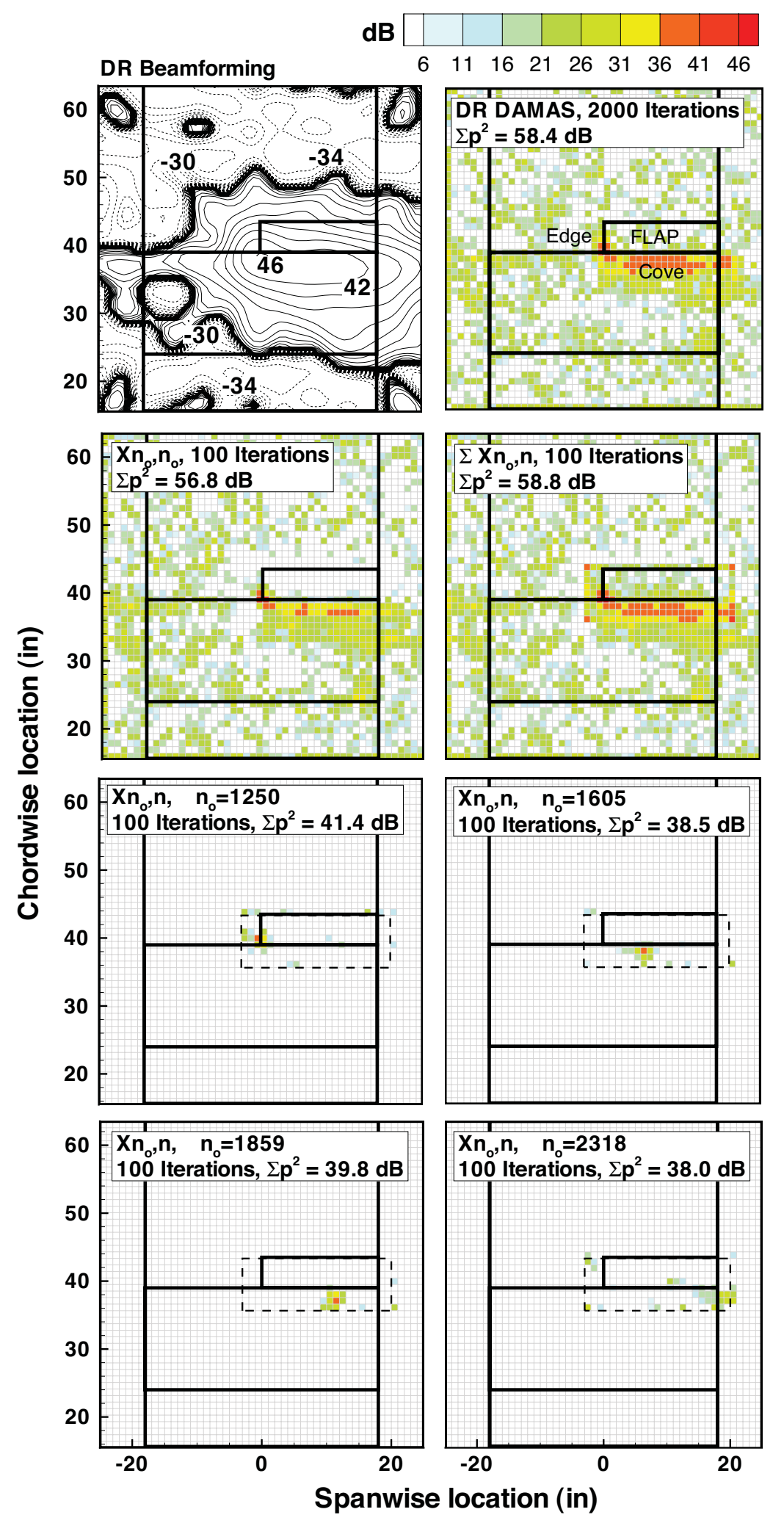

Figure 12. Flap noise test. . Beamforming (top left frame), DAMAS (top right frame), and DAMAS-C (last 6 frames) results for results. $f_{1 / 3}=20 \mathbf{~ k H z}$. 
It should be mentioned that this particular experimental case has elevated spectral levels in this $20 \mathrm{kHz}$ range. The noise is likely due to an aeroacoustic feedback effect or acoustic resonant effect related to the flap gap and flow. Coherent noise regions are expected - likely to be viewed, with respect to the array, as disturbances acting over the gap near the flap leading edge. But with the acoustic wavelength at about .7 inches (corresponding to $20 \mathrm{kHz}$ ), and associated coherent noise source disturbance sizes likely to be less than that, it should not be expected that such regions be discernable with this array's beamwidth at $B_{\text {auto }}=7$ inches. This would be true even if the number of DAMAS-C iterations were substantially increased. A larger array, with a correspondingly smaller beamwidth, would be required. But with this smaller beamwidth, computational efficiency requirements for DAMAS-C would also be higher because of the larger number of grid points required compared to that of the present application due to the resolution requirements such as Eqs. (43) and (44).

\section{Conclusions}

An extension to DAMAS post processing methodology is presented that permits the identification and quantification of coherent sources. DAMAS-C solves this expanded problem using the same basic inverse problem and solution iteration approach as DAMAS. The computational demands are high but DAMAS-C can solve problems not approachable by other methods. Calculations performed for this paper are directed primarily at validating the basic DAMAS-C approach to separate and quantify coherent and incoherent source distributions. This is clearly accomplished. Also, its practical usefulness is shown by an experimental application that reveals that a flap and cove configuration radiates noise in a spatially incoherent manner (within resolution limits) across the cove region. DAMAS-C adds important capabilities to DAMAS. Future ease of use will depend on problem size reduction (see Computational Notes section) and establishing more optimum spatial resolution and iteration criteria (including constraint evaluation). Ultimately, much more significant code speedup (such as through methodologies of Dougherty ${ }^{4}$ ) will be needed to simplify DAMAS-C application and expand evaluation ranges. Still, the capability has been demonstrated and is presently functional.

\section{References}

1. T.F. Brooks and W.M. Humphreys, Jr., A Deconvolution Approach for the Mapping of Acoustic Sources (DAMAS) Determined from Phases Microphone Arrays, $10^{\text {th }}$ AIAA/CEAS Aeroacoustics Conference, Manchester, UK, May 10-12, 2004, AIAA-2004-2954.

2. T.F. Brooks and W.M. Humphreys, Jr., Deconvolution Approach for the Mapping of Acoustic Sources (DAMAS), NASA Invention Disclosure, LAR-16907-1, NASA Langley Research Center, Hampton, VA, April 2004.

3. T.F. Brooks and W.M. Humphreys, Jr., Three-Dimensional Application of DAMAS Methodology for Aeroacoustic Noise Source Definition, $11^{\text {th }}$ AIAA/CEAS Aeroacoustics Conference, Monterey, CA, May 23-25, 2005, AIAA-2005-2960.

4. R.P. Dougherty, Extensions of DAMAS and Benefits and Limitations of Deconvolution in Beamforming, $11^{\text {th }}$ AIAA/CEAS Aeroacoustics Conference, Monterey, CA, May 23-25, 2005, AIAA-2005-2961.

5. R.P. Dougherty, Deconvolution Approach for the Mapping of Acoustic Sources (DAMAS) Applied to Enhance Turbofan Engine Duct Mode Measurements, NOISE-CON 2005, Minneapolis, MN, Oct 17-19.

6. T.F. Brooks and W.M. Humphreys, Jr., Effect of Directional Array Size on the Measurement of Airframe Noise Components, $5^{\text {th }}$ AIAA/CEAS Aeroacoustics Conference, Bellevue, WA, May 10-12, 1999, AIAA-99-1958.

7. W.C. Horne, J.A. Hayes, S.M. Jaeger, and S. Jovic, Effects of Distributed Source Coherence on the Response of Phased Acoustic Arrays, $6^{\text {th }}$ AIAA/CEAS Aeroacoustic Conference, Lahaina, HI, Jun 12-14, 2000, AIAA-20001935. 
8. S. Oerlemans and P. Sijtsma, Determination of Absolute Levels from Phased Array Measurements Using Spatial Source Coherence, , $8^{\text {th }}$ AIAA/CEAS Aeroacoustic Conference, Breckenridge, CO, Jun 17-19, 2002.

9. R.P. Dougherty, Beamforming in Acoustic Testing, Chapter 2 in Aeroacoustic Measurements, Edited by Thomas J. Mueller (Springer, Berlin, 2002), pp. 92-93.

10. T.F. Brooks and W.M. Humphreys, Jr., Extension of DAMAS Phased Array Processing for Spatial Coherence Determination (DAMAS-C), NASA Invention Disclosure”, LAR-17335-1, NASA Langley Research Center, Hampton, VA, April 2006.

11. T.F. Brooks and W.M. Humphreys, Jr., Flap Edge Aeroacoustic Measurements and Predictions, Journal of Sound and Vibration, Vol. 261, pp. 31-74, 2003.

12. J.M. Mendoza, T.F. Brooks, and W.M. Humphreys, Aeroacoustic Measurements of a Leading Edge Slat Configuration, International Journal of Aeroacoustics, pp. 241-274, Vol. 1 (3), 2002. 